\title{
Phylogenetic analysis of infectious bronchitis virus circulating in southern China in 2016-2017 and evaluation of an attenuated strain as a vaccine candidate
}

\author{
Libin Chen ${ }^{1} \cdot$ Bin Xiang ${ }^{1} \cdot$ Yanfen Hong ${ }^{1} \cdot$ Qian $^{\mathrm{Li}^{1}} \cdot$ Haoyun $\mathrm{Du}^{1} \cdot$ Qiuyan Lin ${ }^{1} \cdot$ Ming Liao ${ }^{1} \cdot$ Tao Ren $^{1}$. \\ Chenggang $\mathrm{Xu}^{1}$ (D)
}

Received: 21 March 2020 / Accepted: 5 September 2020 / Published online: 16 October 2020

(c) Springer-Verlag GmbH Austria, part of Springer Nature 2020

\begin{abstract}
Avian infectious bronchitis (IB) is a highly contagious viral respiratory disease, caused by infectious bronchitis virus (IBV), that poses an important economic threat to the poultry industry. In recent years, genotypes GI-7, GI-13, and GI-19 have been the most prevalent IBV strains in China. However, in this study, we found that most IBV strains from southern China in 2016-2017 belonged to genotype GVI-1. This genotype, for which there is no vaccine, has been reported sporadically in the region. The GDTS13 strain, which caused severe IB outbreaks on the farms where it was isolated, was evaluated as a candidate vaccine strain. GDTS13 was serially passaged in specific-pathogen-free embryonated chicken eggs for 100 generations to produce GDTS13-F100. Safety testing indicated that GDTS13-F100 had no pathogenic effect on chickens. Additionally, GDTS13-F100 showed an excellent protective effect against GDTS13, with no clinical signs or virus shedding observed in immunized chickens challenged with the parent strain. These findings indicate that GVI-1 has become the most prevalent IBV genotype in southern China and that GDTS13-F100 may serve as an attenuated vaccine to protect against infection with this genotype.
\end{abstract}

Handling Editor: Sheela Ramamoorthy.

Libin Chen and Bin Xiang have contributed equally to this paper.

Electronic supplementary material The online version of this article (https://doi.org/10.1007/s00705-020-04851-9) contains supplementary material, which is available to authorized users.

Tao Ren

rentao6868@126.com

Chenggang Xu

chgangxu@scau.edu.cn

$1 \quad$ National and Regional Joint Engineering Laboratory for Medicament of Zoonosis Prevention and Control, Key Laboratory of Zoonosis Prevention and Control of Guangdong Province, Key Laboratory of Animal Vaccine Development of Ministry of Agriculture, College of Veterinary Medicine, South China Agricultural University, 483 Wushan Road, Tianhe District, Guangzhou 510642, People's Republic of China

\section{Introduction}

Because of its high morbidity, widespread distribution, and large associated economic losses, avian infectious bronchitis (IB) is considered as one of the most important infectious diseases in the commercial chicken industry $[1,2]$. It is a highly contagious viral respiratory disease caused by infectious bronchitis virus (IBV), which was first described in North Dakota, USA, in the 1930s [3]. In chickens, IBV damages not only the respiratory system but also the bursa of Fabricius, digestive tract tissues, and the urogenital system, resulting in poor egg quality and even death $[1,4]$.

IBV is a coronavirus with an approximately $27-\mathrm{kb}$ positive-sense single-stranded RNA genome that encodes four structural proteins, including the spike glycoprotein (S) and the envelope (E), membrane (M), and nucleocapsid (N) proteins, as well as four non-structural accessory proteins, $3 \mathrm{a}$, $3 \mathrm{~b}, 5 \mathrm{a}$, and $5 \mathrm{~b}$ [5]. The RNA-dependent RNA polymerase of this virus has limited proofreading ability, and mutations and recombination events that occur during replication of the IBV genome lead to the abundant genetic diversity observed for this virus $[6,7]$. Therefore, new genotypes and serotypes of IBV are continually emerging worldwide. 
The S protein has approximately 1170 amino acids and is cleaved into two subunits of nearly equal mass, S1 and S2, after translation [8]. Unlike the conserved S2 subunit, the S1 subunit contains the virus-neutralizing epitope of IBV and is closely associated with the virulence and tissue tropism of the virus $[9,10]$. Nucleotide sequences are most heterogeneous in the $\mathrm{S} 1$ gene, which is prominent in three hypervariable regions (HVRs) (amino acids 38-67, 91-141, and 274-387) [11, 12]. Therefore, to determine the genetic types of IBV, nucleotide sequences of complete or partial S1 genes are commonly analyzed. Among the numerous classifications based on S1 analysis, a new scheme has been proposed that established 35 lineages of IBV, including seven genotypes (GI-GVII), with many additional inter-lineage recombinants $[13,14]$.

Since it was first isolated and identified in 1982, circulation of IBV has been observed in China [15]. Although vaccines based on the 4/91 strain or Massachusetts (Mass) strains such as H120 and H52 have been used for many years, IB continues to be a serious threat to China's poultry industry [16, 17]. In recent years, genotypes GI-7, GI-13, and GI-19, also known as TW-type, 4/91-type, and QX/LX4-type viruses, respectively, have been the major IBV strains isolated in China [17-19]. However, multiple genotypes of IBV, including GVI-1, have been detected sporadically across the country [20]. Specifically, genotype GVI-1 has been isolated in South Korea and China since 2007 and is a respiratory pathogen according to available data [21, 22]. Most GVI-1 strains isolated in China are concentrated in the southern region $[18,21]$.

In this study, 16 IBV strains were isolated from tissue samples of chickens in Guangdong province of southern China in 2016-2017. Phylogenetic and genetic analysis showed that 10 of the isolates belong to genotype GVI-1, indicating that it has been the main genotype of IBV circulating in Guangdong in recent years. We chose one strain, named GDTS13, that caused severe IB outbreaks on farms where it was isolated, for evaluation as a candidate vaccine strain. The GDTS13 strain was attenuated by continuous passage for 100 generations in specific-pathogen-free (SPF) embryonated chicken eggs. Finally, we evaluated the safety of attenuated GVI-1 IBV GDTS13F100 and the protective efficacy of this strain against challenge with various GVI-1 IBV strains. Most tests were performed in accordance with the requirements of the Veterinary Pharmacopoeia of the People's Republic of China (CVP) 2010 Edition and OIE standards (https://www.oie.int/fileadmin/Home/eng/ Health_standards/tahm/3.03.02_AIB.pdf).

\section{Materials and methods}

\section{Embryonated chicken eggs and chicks used in experiments}

Embryonated SPF White Leghorn eggs and chicks were purchased from Guangdong Wens Dahuanong Biotechnology Co., Ltd. (Guangzhou, China). All animal experiments were conducted under the guidance of the South China Agricultural University Institutional Animal Care and Use Committee and Association for Assessment and the Accreditation of Laboratory Animal Care International-Accredited Facilities. The protocol was reviewed and approved by the Committee on the Ethics of Animal Experiments of South China Agricultural University (approval ID no. SCXK (Guangdong) 2018-0027).

\section{Virus isolation and RNA extraction}

Sixty-two tissue samples, including the kidneys, tracheas, and lungs, were collected from broiler chickens suspected of being infected with IBV on poultry farms in Guangdong province in 2016-2017. These chickens exhibited clinical signs, including lethargy, feather ruffling, neurologic symptoms, and sneezing. All of the sick chickens had been immunized against Mass strains such as H120 and H52. The samples were cut and ground, frozen and thawed three times, suspended in sterile phosphate-buffered saline (PBS) containing $200 \mathrm{U} / \mathrm{mL}$ penicillin and $200 \mu \mathrm{g} / \mathrm{mL}$ streptomycin, and then centrifuged at $10,000 \mathrm{rpm}$ for $5 \mathrm{~min}$ at $4^{\circ} \mathrm{C}$. The supernatants were collected and inoculated into 10-dayold SPF chicken embryonated eggs by the allantoic cavity route. Embryos that died within $24 \mathrm{~h}$ after inoculation were discarded. Infectious allantoic fluid was collected at $60 \mathrm{~h}$ post-inoculation and stored at $-80^{\circ} \mathrm{C}$ until further processing. RNA was extracted from the allantoic fluid using a RaPure Viral RNA/DNA Kit (Magen, Guangdong, China) according to the manufacturer's instructions. IBV infection was verified by reverse transcription polymerase chain reaction (RTPCR) using a PrimeScript One Step RT-PCR Kit (Takara, Shiga, Japan) for the M gene. Passages GDTS13-F40, F60, F80, and F100 were obtained by continuous passage of GDTS13 through 10-day-old SPF chicken embryonated eggs for $40,60,80$, and 100 passages, respectively. The median embryo infectious doses $\left(\mathrm{EID}_{50}\right)$ of the strains were calculated as described by Reed and Muench [23].

\section{RT-PCR and sequencing}

Pairs of primers targeting the $\mathrm{S} 1$ gene and 17 fragments of the complete IBV genome (Supplementary Table 1) were 
designed to amplify, clone, and sequence the genomes of isolates using Oligo 7. The genomic sequence of the GDTS13 strain and sequences of the S1 gene of other isolates were verified by RT-PCR using a PrimeScript One Step RT-PCR Kit Ver. 2 (Takara). Target products obtained by RT-PCR were purified and recovered using a Gel Extraction Kit (Omega Bio-tek, Norcross, GA, USA). The RT-PCR products were ligated into the pMD19-T vector (Takara) and used to transform DH5 $\alpha$ competent cells (Tiangen, Beijing, China). Plasmids positive for these products were sequenced by Sangon Biotech (Shanghai) Co., Ltd. (Shanghai, China).

\section{Genetic and phylogenetic analysis of the $\mathrm{S} 1$ gene}

All IBV S1 gene nucleotide and protein sequences were aligned using EditSeq (DNASTAR, Madison, WI, USA) and compared with 156 reference IBV strains $(\sim 1620$ bp) representing 35 well-established lineages and seven genotypes published in GenBank, including all 49 strains of the GVI-1 genotype (updated March 1, 2020), using the ClustalW multiple alignment algorithm in MegAlign (DNASTAR). S1 gene nucleotide sequences were phylogenetically analyzed using the maximum-likelihood method in MEGA version 10.0.2 (https://www.megasoftware.net) and the Tamura-Nei model with 1000 bootstrap replicates.

\section{Virus cross-neutralization testing}

Immune sera were prepared as described previously [24] against vaccine strains H120, 4/91, M41, Holte, and Conn46. The immune sera were serially diluted twofold with sterile PBS, and each dilution was mixed with an equal volume of a suspension containing $200 \mathrm{EID}_{50}$ of each strain. The mixtures were incubated at $37^{\circ} \mathrm{C}$ for $1 \mathrm{~h}$. Ten-day-old SPF chicken embryonated eggs were inoculated with the mixture via the allantoic cavity route and incubated at $37^{\circ} \mathrm{C}$ for 7 days. The serum neutralization titer was the highest dilution of serum that protected more than $50 \%$ of the chicken embryos dwarfing or death following vaccine challenge.

\section{Evaluation of GDTS13 strain pathogenicity in SPF chickens}

Allantoic fluid containing GDTS13 was tested for other pathogens including bacteria, mycoplasma, Newcastle disease virus, avian influenza virus, infectious laryngotracheitis virus, avian reovirus, infectious bursal disease virus, Marek's disease virus, avian reticuloendothelial hyperplasia virus, avian lymphoblastic leukemia virus, avian encephalomyelitis virus, chicken pox virus, and avian adenovirus. To evaluate the pathogenicity of the GDTS13 strain, 100 1-day-old SPF chickens were randomly divided into two groups $(\mathrm{n}=50)$, with chicks in one group challenged with allantoic fluid containing the GDTS13 strain at a dose of $10^{5} \mathrm{EID}_{50} / 200 \mu \mathrm{L}$ per chick via the intraocular $(100 \mu \mathrm{L})$ and intranasal $(100 \mu \mathrm{L})$ routes and 50 chicks inoculated with $200 \mu \mathrm{L}$ of sterile PBS via the same routes as controls. Ten chicks in each group were randomly selected and sacrificed on days 3, 5, 7, and 9 postinfection. Trachea, lung, kidney, and glandular stomach tissues were collected from the chickens and ground for virus isolation as described above. All chickens were maintained in isolators under negative pressure and observed daily for illness or death throughout the experimental period. Any chick showing clinical signs was considered diseased.

\section{Safety testing of different generations of the GDTS13 strain}

Fifty 1-day-old SPF chickens were randomly divided into five groups $(\mathrm{n}=10)$, four of which (groups $\mathrm{A}, \mathrm{B}, \mathrm{C}$, and $\mathrm{D})$ were challenged with allantoic fluid containing the GDTS13 strain at a dose of $10^{5} \mathrm{EID}_{50} / 200 \mu \mathrm{L}$ per chick at various passages (GDTS13-F40, -F60, -F80, and -F100, respectively) via the intraocular and intranasal routes. Ten chicks were inoculated with $200 \mu \mathrm{L}$ of sterile PBS via the same route as a control group (group E). All chicks were sacrificed at 15 days postinfection, and the trachea, lung, kidney, and glandular stomach tissues were collected and ground for virus isolation. All chicks were maintained in isolators under negative pressure and observed daily for illness or death throughout the experimental period. Any chick showing clinical signs was considered diseased.

\section{Determination of the protective efficacy of GDTS13-F100 against challenge with genotype GVI-I field strains}

Forty 1-day-old SPF chickens were randomly divided into four groups of 10 chickens each and housed in different isolators. Chicks in group A were inoculated with GDTS13-F100 at a dose of $10^{3.5} \operatorname{EID}_{50} / 100 \mu \mathrm{L}$ per chick via the intraocular and intranasal routes. Chicks in group $\mathrm{B}$ were intraocularly and intranasally vaccinated with one dose of H120 (Boehringer Ingelheim, Ingelheim am Rhein, Germany) as recommended by the manufacturer. Chicks in groups $\mathrm{C}$ and D were inoculated with $100 \mu \mathrm{L}$ of sterile PBS via the same routes. At 14 days post-immunization, chicks in groups $\mathrm{A}, \mathrm{B}$, and $\mathrm{C}$ were challenged with allantoic fluid containing the GDTS13 strain at a dose of $10^{5} \mathrm{EID}_{50} / 200 \mu \mathrm{L}$ per chick via the intraocular and intranasal routes, whereas chicks in group D were inoculated with $200 \mu \mathrm{L}$ of sterile PBS via the same route to as controls. All chicks were observed daily for death and clinical signs attributable to IBV infection for 14 days. Any chick showing clinical signs was considered diseased. Oropharyngeal and cloacal swabs 
were obtained at $3,5,7$, and 9 days postinfection to detect IBV shedding from chickens. Swabs were placed in $1 \mathrm{~mL}$ of sterile PBS containing $200 \mathrm{U}$ of penicillin and $200 \mu \mathrm{g}$ of streptomycin per $\mathrm{mL}$, and each fluid was inoculated into five SPF chicken embryonated eggs. The allantoic fluid was tested for IBV by RT-PCR as described above.

\section{Results}

\section{Virus isolation}

Sixty-two tissue samples were collected from chickens suspected of being infected with IBV on poultry farms in Guangdong province in 2016-2017. As shown in Fig. 1, 16 IBV strains were isolated from the tissue samples of chickens obtained in different cities. Huizhou showed the highest rate of isolation from tissue samples $(9 / 20,45 \%)$, followed by Yunfu $(2 / 6,33.3 \%)$ and Jiangmen (5/18, 27.8\%).
IBV was not isolated from tissue samples from Qingyuan (0/6), Foshan (0/4), or Guangzhou (0/8). Supplementary Table $\mathrm{S} 2$ shows information on each isolated IBV strain.

\section{Phylogenetic analysis and sequence comparison based on complete S1 genes of 16 IBV strains}

As shown in Figure 2A, one of the IBV isolates (TJ402) clustered with the GI-13 lineage of IBV, and four isolates (TJ401, GDJM502, GDJM505, and GDSB1220) were classified as lineage GI-19, which includes the QX-type strain, the main epidemic strain circulating in China in recent years. Additionally, 10 of the IBV isolates belonged to lineage GVI-1, which comprises isolates collected in China and Korea after 2007. Combined with the data of this study, the majority of the GVI-1 strains published in GenBank were isolated in south China $(34 / 59,57.6 \%)$ (Fig 2B).

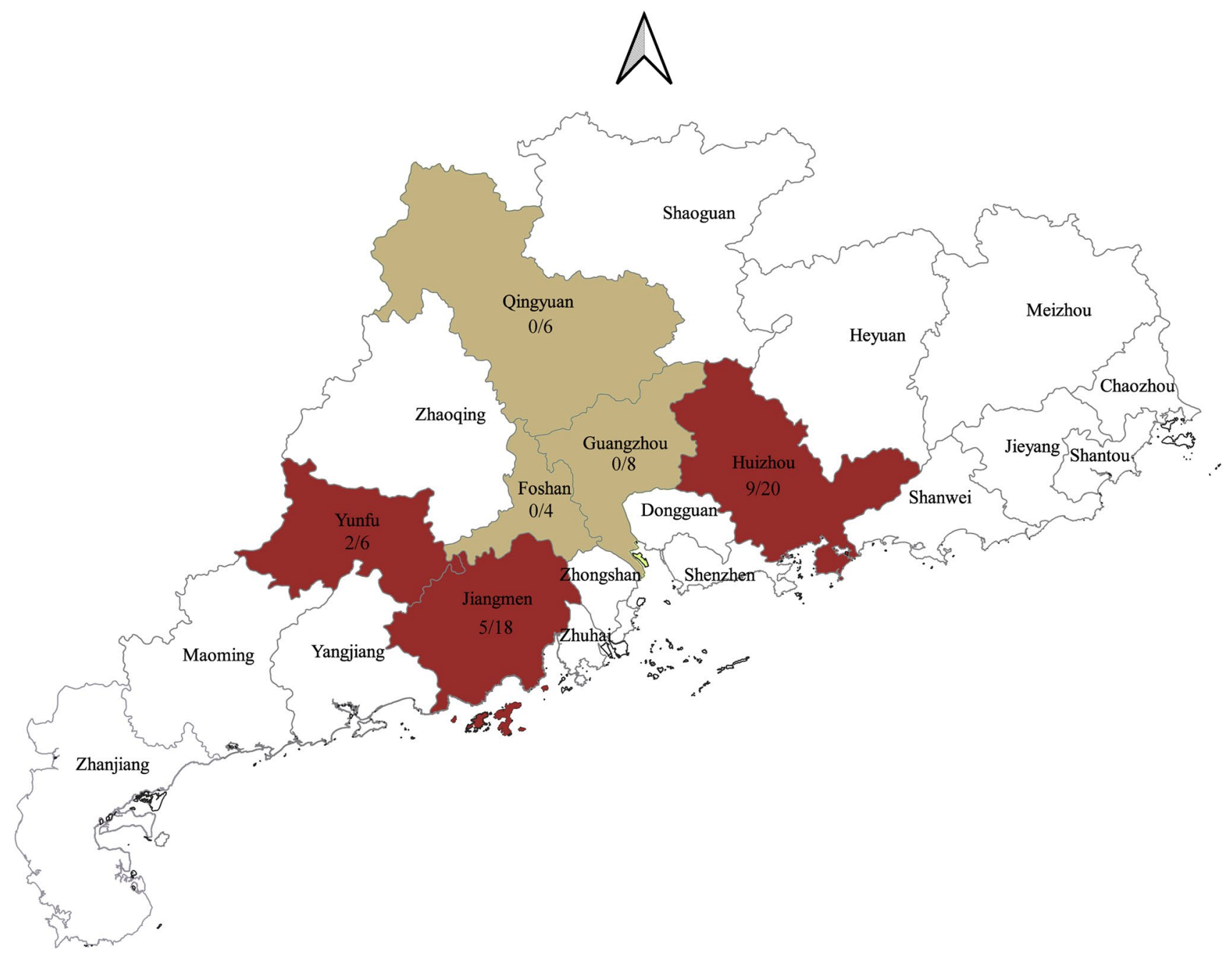

Fig. 1 Sources of IBV isolates from Guangdong province 
A

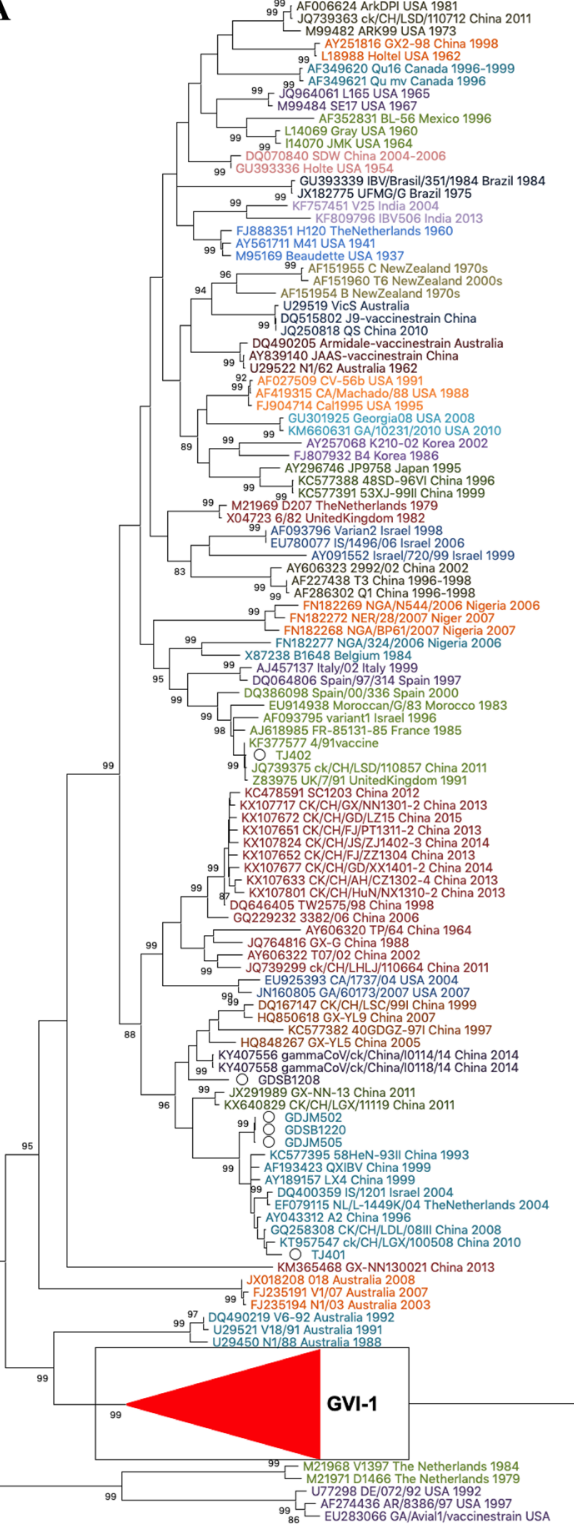

te-9

GI-4

GI-20

GI-8

GI-3

GI-2

GI-11

GI-1

GI-10

GI-6

GI-5

GI-17

GI-27

GI-15

GI-18

GI-12

GI-23

GI-16

GI-26

GI-14

GI-21

GI-13

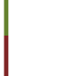

G1-7

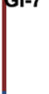

GI-25

GI-22

G1-29

GI-28

GI-19

Gl-19

GVII-1

GIII-1

GIIl-1

GIV-1

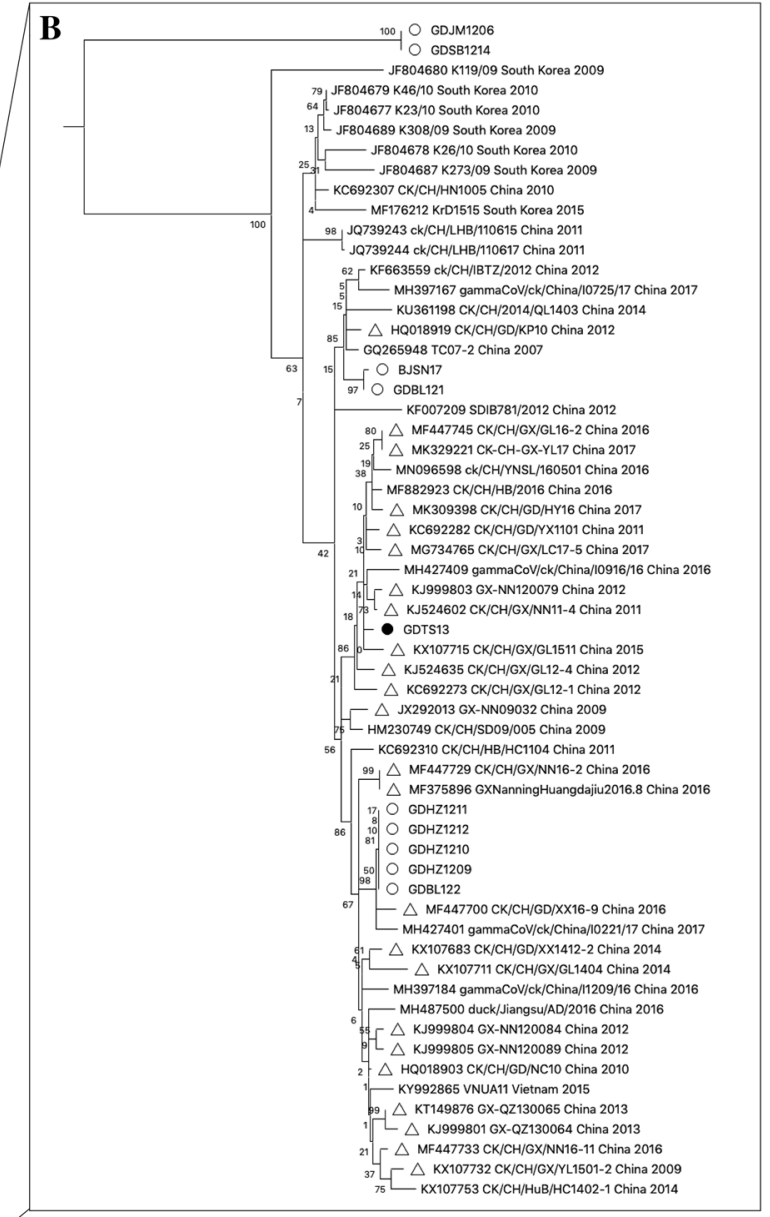

Fig. 2 Phylogenetic relationships between 16 isolates and 156 reference strains based on complete S1 gene sequences. The tree was created by the maximum-likelihood method in MEGA version 10.0.2 using the Tamura-Nei model and 1000 bootstrap replicates. Twentyfour reference GVI-1 strains from southern China are indicated by

\section{Analysis of S1 structural proteins}

The GDTS13-F100 S1 gene was found to contain four mutations resulting in three amino acid changes (Table 1). The amino acid substitution R289K was located in HVR2 of the $\mathrm{S} 1$ protein. The nucleotide and amino acid sequences of GDTS13-F80 and GDTS13-F100 were both 100\% identical, indicating that GDTS13 was genetically stable after serial passage in SPF chicken embryonated eggs. triangles, whereas the 15 isolates from this study are indicated by open circles, and GDTS13 is indicated by a solid circle. (A) Strains of the same lineages are shown in the same color. (B) Ten isolates are shown with all GVI-1 strain sequences (49 isolates) published in GenBank (updated to March 1, 2020).

\section{Antigenic characterization of strain GDTS13}

Strain GDTS13, one of the 10 isolates classified as belonging to lineage GVI-1, caused a severe IB outbreak on farms where it was isolated. After isolation and purification, no other pathogens were detected in the allantoic fluid containing strain GDTS13. To evaluate cross-neutralization reactivity between GDTS13 and vaccines that are widely used in China, five vaccine strains (H120, 4/91, Conn46, M41, 
and Holte) and GDTS13 were used to prepare immune sera. Serum antibodies against strain GDTS13 and five vaccine strains showed a high titer, suggesting that these sera were appropriate for use in a neutralization test. As shown in Table 2, the neutralization titers of antibodies against the five vaccine strains were all less than 8 against strain GDTS13, indicating that serum antibodies produced in response to the vaccine strains did not effectively neutralize this strain.

\section{Evaluation of the pathogenicity of strain GDTS13 in SPF chickens}

All chickens in the GDTS13-infection groups exhibited previously described clinical manifestations of IBV [17, 25, 26], including lethargy, feather ruffling, neck shrinkage, head shaking, sneezing, and diarrhea. GDTS13 virus replicated in the trachea, lung, kidney, and glandular stomach tissues from days 3 to 9 postinfection, whereas the virus was only detected in the liver tissue from days 3 to 5 postinfection. Replication of strain GDTS13 was most obvious in the trachea and lungs (Fig 3).

\section{Safety testing of different passages of strain GDTS13}

To evaluate whether the pathogenicity of GDTS13 was attenuated by serial passaging, SPF chickens were challenged with the GDTS13 strain from different passages. Fifty percent of SPF chickens inoculated with GDTS13F40 exhibited clinical manifestations, including lethargy, feather ruffling, neck shrinkage, head shaking, and sneezing, whereas only $20 \%$ of SPF chicken inoculated with
Table 1 Amino acid changes observed in the S1 protein of different generations of IBV genotype GVI-1 strain GDTS13

Table 2 Virus crossneutralization testing with GDTS13 and other IBV vaccine strains

Fig. 3 Virus recovery from different tissues after infection with strain GDTS13.

\begin{tabular}{|c|c|c|c|c|c|c|c|c|c|c|c|c|c|c|c|c|c|c|c|c|c|}
\hline \multirow[b]{2}{*}{ GDTS13-F1 } & \multicolumn{7}{|c|}{33} & \multicolumn{7}{|c|}{289} & \multicolumn{7}{|c|}{397} \\
\hline & $\mathrm{Y}$ & $\mathrm{Y}$ & $\mathrm{Y}$ & $\mathbf{Q}$ & $\mathrm{S}$ & G & $\mathrm{F}$ & $\mathrm{P}$ & $\mathrm{N}$ & Q & $\mathbf{R}$ & $\mathrm{E}$ & $\mathrm{P}$ & $\mathrm{S}$ & $\mathrm{Y}$ & $\mathrm{S}$ & $\mathrm{G}$ & D & $\mathrm{L}$ & $\mathrm{N}$ & Q \\
\hline GDTS13-F20 & $\mathrm{Y}$ & $\mathrm{Y}$ & $\mathrm{Y}$ & $\mathbf{Q}$ & $\mathrm{S}$ & G & $\mathrm{F}$ & $\mathrm{P}$ & $\mathrm{N}$ & Q & $\mathbf{R}$ & $\mathrm{E}$ & $\mathrm{P}$ & $\mathrm{S}$ & $\mathrm{Y}$ & $\mathrm{S}$ & G & $\mathbf{N}$ & $\mathrm{L}$ & $\mathrm{N}$ & Q \\
\hline GDTS13-F40 & $\mathrm{Y}$ & $\mathrm{Y}$ & $\mathrm{Y}$ & $\mathbf{Q}$ & $\mathrm{S}$ & G & $\mathrm{F}$ & $\mathrm{P}$ & $\mathrm{N}$ & Q & $\mathbf{R}$ & $\mathrm{E}$ & $\mathrm{P}$ & $\mathrm{S}$ & $\mathrm{Y}$ & $\mathrm{S}$ & G & $\mathbf{N}$ & $\mathrm{L}$ & $\mathrm{N}$ & Q \\
\hline GDTS13-F60 & $\mathrm{Y}$ & $\mathrm{Y}$ & $\mathrm{Y}$ & $\mathbf{Q}$ & $\mathrm{S}$ & G & $\mathrm{F}$ & $\mathrm{P}$ & $\mathrm{N}$ & Q & $\mathbf{K}$ & $\mathrm{E}$ & $\mathrm{P}$ & $\mathrm{S}$ & $\mathrm{Y}$ & $\mathrm{S}$ & G & $\mathbf{N}$ & $\mathrm{L}$ & $\mathrm{N}$ & Q \\
\hline GDTS13-F80 & $\mathrm{Y}$ & $\mathrm{Y}$ & $\mathrm{Y}$ & $\mathbf{K}$ & $\mathrm{S}$ & G & $\mathrm{F}$ & $\mathrm{P}$ & $\mathrm{N}$ & Q & $\mathbf{K}$ & $\mathrm{E}$ & $\mathrm{P}$ & $\mathrm{S}$ & $\mathrm{Y}$ & $\mathrm{S}$ & G & $\mathbf{N}$ & $\mathrm{L}$ & $\mathrm{N}$ & Q \\
\hline GDTS13-F100 & $\mathrm{Y}$ & $\mathrm{Y}$ & $\mathrm{Y}$ & $\mathbf{K}$ & $\mathrm{S}$ & G & $\mathrm{F}$ & $\mathrm{P}$ & $\mathrm{N}$ & Q & $\mathbf{K}$ & $\mathrm{E}$ & $\mathrm{P}$ & $\mathrm{S}$ & $\mathrm{Y}$ & $\mathrm{S}$ & G & $\mathbf{N}$ & & $\mathrm{N}$ & Q \\
\hline
\end{tabular}

\begin{tabular}{|c|c|c|c|c|c|c|}
\hline \multirow[t]{2}{*}{ Strain } & \multirow[t]{2}{*}{$4 / 91$} & \multicolumn{2}{|c|}{ Massachusetts } & \multirow[t]{2}{*}{ Holte } & \multirow[t]{2}{*}{ Conn46 } & \multirow[t]{2}{*}{ GDTS13 } \\
\hline & & H120 & M41 & & & \\
\hline $4 / 91$ & 64 & - & - & - & - & - \\
\hline H120 & - & 32 & - & - & - & - \\
\hline M41 & - & - & 64 & - & - & - \\
\hline Holte & - & - & - & 64 & - & - \\
\hline Conn46 & - & - & - & - & 128 & - \\
\hline GDTS13 & 0 & 2 & 4 & 4 & 2 & 64 \\
\hline
\end{tabular}

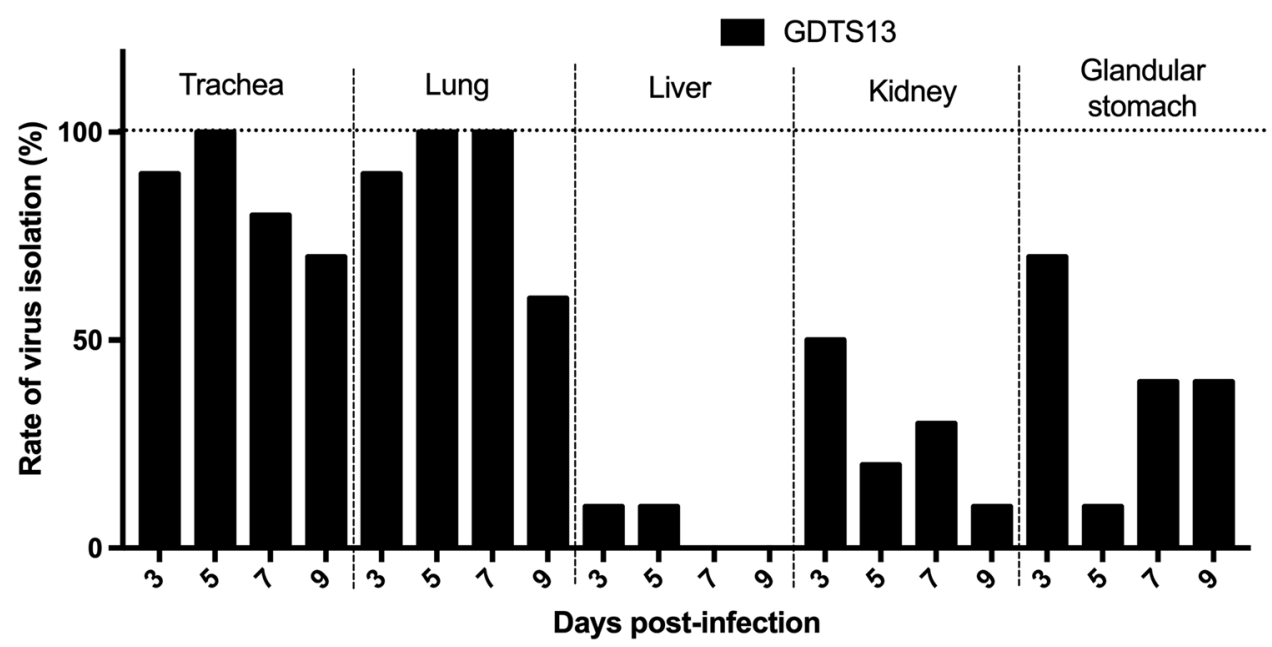


GDTS13-F60 exhibited these signs. No clinical signs were observed in SPF chickens inoculated with GDTS13-F80 or GDTS13-F100 for 15 days after infection.

All chickens were sacrificed and dissected at 15 days postinfection. The percentages of SPF chickens challenged with strains GDTS13-F40 and GDTS13-F60 that showed disease were $60 \%$ and $10 \%$, respectively. Macroscopic lesions mainly manifested as tracheal and laryngeal bleeding, increased mucus, bleeding spots in the liver, and, in some chickens, kidney enlargement. No clinical signs or pathological changes were observed in chickens inoculated with GDTS13-F80 or GDTS13-F100.

\section{GDTS13-F100 as a vaccine candidate against IBV in southern China}

To evaluate the potential for GDTS13-F100 to serve as an attenuated vaccine, we compared the protective effect of F100 and H120 against challenge with the GDTS13 strain. The morbidity of chickens inoculated with GDTS13 was 90\%. In the GDTS13-F100-inoculated groups, no chickens challenged with GDTS13 showed clinical manifestations. However, in the H120-inoculated group, the morbidity of chickens challenged with GDTS13 was 50\%. Analysis of oropharyngeal and cloacal swabs showed that all IBV-inoculated chickens in the control groups shed IBV between 3 and 9 days postinfection. No GDTS13-challenged chickens in the GDTS13-F100-immunized group shed virus between 3 and 9 days postinfection, whereas $70 \%$ of GDTS13-challenged chickens in the H120-immunized group shed virus between 5 and 9 days postinfection (Fig. 4).

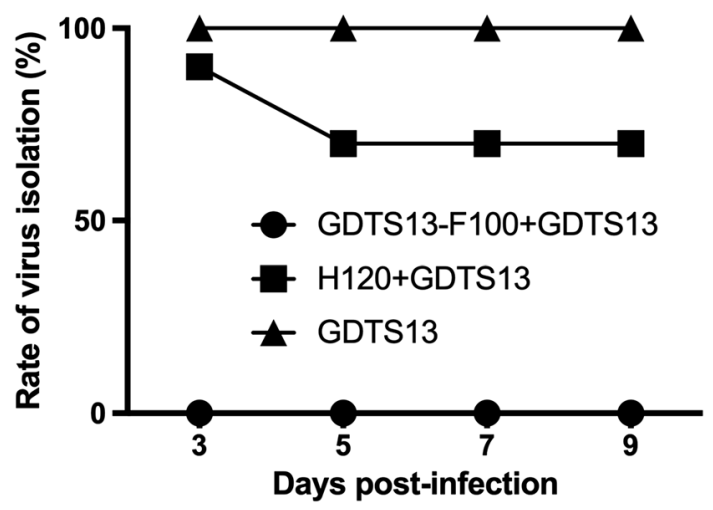

Fig. 4 Virus recovery from oropharyngeal and cloacal swabs from chickens challenged with GDTS13 and vaccine strains of IBV

\section{Discussion}

Guangdong province in southern China is considered a reservoir for poultry viruses. This region has a large number of free-range poultry farms and plays a vital role in bird migration, providing ideal conditions for recombination and transmission of avian viruses, including avian influenza virus (AIV) and IBV. Mixed infections of IBV and low-pathogenic AIV can cause severe illness and high mortality, and result in large economic losses for poultry farms [27]. Although vaccines based on the Mass-type and 4/91 strains of IBV have been used for many years in the poultry industry, IB continues to be prevalent in China [15, 28, 29]. Therefore, epidemiological surveillance of avian viruses in Guangdong province is important for protecting the poultry industry in southern China and can provide a theoretical basis for designing a program to prevent and control avian diseases.

IBV is classified into serotypes and genotypes based on the characteristics of the S gene and protein [30]. In this study, we used a new classification scheme to type IBV based on the complete $\mathrm{S} 1$ gene sequence $[13,14]$. In China, the epidemiology of IBV is complex because of the vast area of virus circulation [15]. Viruses of genotypes GI-7 (4/91-type), GI-13 (TW-type), and GI-19 (QX/LX4type) have been the ones isolated most frequently in recent years [18, 19], whereas other genotypes are predominant elsewhere in China. For example, from 2011 to 2015, the IBV strains that were dominant in central and eastern China belonged to genotype GI-19, whereas those in southern China were GI-7 [18, 19]. In this study, 16 strains were isolated from 62 tissue samples, showing a positive rate similar to those reported in previous studies [17, 31]. However, in this study, 10 of 16 isolates from Guangdong province in 2016-2017 were classified as genotype GVI1; only five isolates were classified as GI-19. Our results indicate that genotype GVI-1 IBV is the dominant epidemic strain in Guangdong. Notably, all of the chickens from which isolates were obtained had been immunized with the Mass-type vaccine, but they still displayed clinical signs of IB, suggesting that the Mass-type vaccine used in the poultry industry in China did not effectively prevent infection with genotype GVI-1 IBV strains. Genotype GVI-1 IBV strains have been isolated from chickens in Korea and China since 2007 and were originally classified as 'Korean new cluster II' [22] or 'Chinese new-type' [32] IBVs. Increasingly, however, TC07-2-type genotype GVI strains of IBV have been reported in southern China $[18,33]$. The sequences of all GVI-1 strains published in GenBank were downloaded for phylogenetic analysis, and the results indicated that the majority of GVI-1 strains were isolated from southern China, which is consistent 
with previous studies $[18,20]$. Although there are no data available for GVI-1 in China after 2017, the spread of GVI in southern China should not be ignored. Serotype differences among genetically distinct IBVs generally correlate with variations in the HVRs of the S1 protein gene [11, $34]$, and differences as low as 5\% between $\mathrm{S} 1$ sequences of IBV can result in poor cross-protection by vaccines currently in use [15, 35]. Because of the large differences between the S1 protein amino acid sequences of the Masstype vaccine and GVI-1 strains, infection with GVI-1 IBVs cannot be effectively prevented, and strains of this genotype spread easily through unprotected populations. Therefore, the spread of genotype GVI-1 IBV in southern China urgently requires attention, and more-effective vaccines are needed to limit the circulation of these viruses.

Because of the potential threat posed by GVI-1 IBV to southern China and the lack of a vaccine for protecting chickens against infection with this genotype, we evaluated a newly isolated strain for use as a GVI-1 IBV vaccine. One of these isolates, GDTS13, induced typical signs of IB in 1-day-old SPF chickens, including lethargy, feather ruffling, neck shrinkage, head shaking, sneezing, and diarrhea. Autopsy revealed tracheal, laryngeal, and liver hemorrhage, excessive mucus, and kidney enlargement, and IBV was isolated from the trachea, lungs, kidneys, liver, and glandular stomach. However, the amount of virus in the liver was relatively low, suggesting that this organ is not the major target of strain GDTS13. Furthermore, cross-neutralization testing showed that immune sera against the five vaccine strains (Holte, Conn46, M41, H120, and 4/91) did not effectively neutralize strain GDTS13, indicating that none of the vaccines currently in use can effectively prevent infection with genotype GVI-1 IBV. This may account, at least in part, for the increased incidence of infection with genotype GVI-1 strains in southern China in recent years and explain why genotype GVI-1 was isolated from immunized chickens.

Continuous passage in embryonated SPF chicken eggs is the conventional method for developing attenuated IBV vaccine strains $[15,36]$, and the pathogenicity of IBV is closely associated with mutations in the $\mathrm{S} 1$ protein variants $[37,38]$. In this study, the S1 gene of GDTS13-F100 was found to contain four mutations resulting in amino acid changes. Alterations in the $\mathrm{S} 1$ protein sequence may be an important reason for the reduced virulence of GDTS13, but this remains to be verified.

According to the OIE standards and requirements of the CVP (2010 Edition) [39], a live vaccine is suitable for use if at least $80 \%$ of challenge-vaccinated birds show no evidence of IBV in their trachea, whereas $80 \%$ or more of the control birds should have evidence of the presence of the virus. In this study, GDTS13-F100 elicited a more effective immune response (100\% protection) than $\mathrm{H} 120$ against IBV genotype GVI-1, whereas all chickens in the control group shed IBV after infection with the GDTS13 strain. Because genotype GVI-1 strains are prevalent in southern China and the widely used Mass-type vaccines cannot protect against them, GDTS13-F100 may be useful as an attenuated vaccine to prevent infection with GVI-1 strains and reduce economic losses due to IB. However, whether the GDTS13 vaccine broadly protects against more-heterologous IBV strains require further analysis.

Acknowledgements This study was supported by the National Key Research and Development Program of China (nos. 2018YFD0500100 and 2016YFD0501603), the National Natural Science Foundation of China (no. 31872492), and the Poultry Production Technology of Guangdong System (no. 2018LM1115).

\section{Compliance with ethical standards}

Conflict of interest The authors declare no conflict of interest.

\section{References}

1. Cavanagh D (2007) Coronavirus avian infectious bronchitis virus. Vet Res 38(2):281-297. https://doi.org/10.1051/vetres:2006055

2. Jackwood MW (2012) Review of infectious bronchitis virus around the world. Avian Dis 56(4):634-641. https://doi. org/10.1637/10227-043012-Review.1

3. Schalk AF, Hawn MC (1931) An apparently new respiratory disease of baby chicks. Jamvetmedassoc 78:413-422

4. Johnson RB, Marquardt WW (1975) The neutralizing characteristics of strains of infectious bronchitis virus as measured by the constant-virus variable-serum method in chicken tracheal cultures. Avian Dis 19(1):82-90

5. Boursnell ME, Brown TD, Foulds IJ, Green PF, Tomley FM, Binns MM (1987) Completion of the sequence of the genome of the coronavirus avian infectious bronchitis virus. J Gen Virol 68(Pt 1):57-77. https://doi.org/10.1099/0022-1317-68-1-57

6. Kottier SA, Cavanagh D, Britton P (1995) Experimental evidence of recombination in coronavirus infectious bronchitis virus. Virology 213(2):569-580. https://doi.org/10.1006/viro.1995.0029

7. Shi Q, Wang C, Keirs RW (2000) Genetic relationships of infectious bronchitis virus isolates from Mississippi broilers. Avian Dis 44(1):66-73

8. Cavanagh D (1983) Coronavirus IBV glycopolypeptides: size of their polypeptide moieties and nature of their oligosaccharides. J Gen Virol 64(Pt 5):1187-1191. https://doi. org/10.1099/0022-1317-64-5-1187

9. Cavanagh D, Davis PJ (1986) Coronavirus IBV: removal of spike glycopolypeptide $\mathrm{S} 1$ by urea abolishes infectivity and haemagglutination but not attachment to cells. J Gen Virol 67(Pt 7):14431448. https://doi.org/10.1099/0022-1317-67-7-1443

10. Ismail MM, Cho KO, Hasoksuz M, Saif LJ, Saif YM (2001) Antigenic and genomic relatedness of turkey-origin coronaviruses, bovine coronaviruses, and infectious bronchitis virus of chickens. Avian Dis 45(4):978-984

11. Cavanagh D, Davis PJ, Mockett AP (1988) Amino acids within hypervariable region 1 of avian coronavirus IBV (Massachusetts serotype) spike glycoprotein are associated with neutralization epitopes. Virus Res 11(2):141-150

12. Moore KM, Jackwood MW, Hilt DA (1997) Identification of amino acids involved in a serotype and neutralization specific 
epitope within the s1 subunit of avian infectious bronchitis virus. Arch Virol 142(11):2249-2256

13. Valastro V, Holmes EC, Britton P, Fusaro A, Jackwood MW, Cattoli G, Monne I (2016) S1 gene-based phylogeny of infectious bronchitis virus: An attempt to harmonize virus classification. Infect Genet Evol 39:349-364. https://doi.org/10.1016/j.meegi d.2016.02.015

14. Ma T, Xu L, Ren M, Shen J, Han Z, Sun J, Zhao Y, Liu S (2019) Novel genotype of infectious bronchitis virus isolated in China. Vet Microbiol 230:178-186. https://doi.org/10.1016/j.vetmi c. 2019.01 .020

15. Liu SW, Zhang QX, Chen JD, Han ZX, Liu X, Feng L, Shao YH, Rong JG, Kong XG, Tong GZ (2006) Genetic diversity of avian infectious bronchitis coronavirus strains isolated in China between 1995 and 2004. Arch Virol 151(6):1133-1148. https:// doi.org/10.1007/s00705-005-0695-6

16. Liu S, Kong X (2004) A new genotype of nephropathogenic infectious bronchitis virus circulating in vaccinated and nonvaccinated flocks in China. Avian Pathol 33(3):321-327. https:// doi.org/10.1080/0307945042000220697

17. Xu L, Han Z, Jiang L, Sun J, Zhao Y, Liu S (2018) Genetic diversity of avian infectious bronchitis virus in China in recent years. Infect Genet Evol 66:82-94. https://doi.org/10.1016/j.meegi d.2018.09.018

18. Feng K, Wang F, Xue Y, Zhou Q, Chen F, Bi Y, Xie Q (2017) Epidemiology and characterization of avian infectious bronchitis virus strains circulating in southern China during the period from 2013-2015. Sci Rep 7(1):6576. https://doi.org/10.1038/s4159 8-017-06987-2

19. Zhao Y, Zhang H, Zhao J, Zhong Q, Jin JH, Zhang GZ (2016) Evolution of infectious bronchitis virus in China over the past two decades. J Gen Virol 97(7):1566-1574. https://doi.org/10.1099/ jgv.0.000464

20. Fan W, Tang N, Dong Z, Chen J, Zhang W, Zhao C, He Y, Li M, Wu C, Wei T, Huang T, Mo M, Wei P (2019) Genetic analysis of avian coronavirus infectious bronchitis virus in yellow chickens in Southern China over the past decade: revealing the changes of genetic diversity, dominant genotypes, and selection pressure. Viruses. https://doi.org/10.3390/v11100898

21. Li L, Xue C, Chen F, Qin J, Xie Q, Bi Y, Cao Y (2010) Isolation and genetic analysis revealed no predominant new strains of avian infectious bronchitis virus circulating in South China during 2004-2008. Vet Microbiol 143(2-4):145-154. https://doi. org/10.1016/j.vetmic.2009.11.022

22. Lim TH, Kim MS, Jang JH, Lee DH, Park JK, Youn HN, Lee JB, Park SY, Choi IS, Song CS (2012) Live attenuated nephropathogenic infectious bronchitis virus vaccine provides broad cross protection against new variant strains. Poult Sci 91(1):89-94. https:// doi.org/10.3382/ps.2011-01739

23. Reed LJ, Muench H (1938) A simple method of estimating fifty per cent endpoints. Am J Epidemiol 27(3):493-497. https://doi. org/10.1093/oxfordjournals.aje.a118408

24. Gao M, Wang Q, Zhao W, Chen Y, Zhang T, Han Z, Xu Q, Kong $X$, Liu S (2016) Serotype, antigenicity, and pathogenicity of a naturally recombinant TW I genotype infectious bronchitis coronavirus in China. Vet Microbiol 191:1-8. https://doi.org/10.1016/j. vetmic.2016.05.018

25. Zhao Y, Xie D, Zhang K, Cheng J, Xu G, Zhang G (2019) Pathogenicity of a GI-22 genotype infectious bronchitis virus isolated in China and protection against it afforded by GI-19 vaccine. Virus Res 267:59-66. https://doi.org/10.1016/j.virusres.2019.05.006
26. Yan S, Zhao J, Xie D, Huang X, Cheng J, Guo Y, Liu C, Ma Z, Yang H, Zhang G (2018) Attenuation, safety, and efficacy of a QX-like infectious bronchitis virus serotype vaccine. Vaccine 36(14):1880-1886. https://doi.org/10.1016/j.vaccine.2018.02.053

27. Hassan KE, Ali A, Shany SAS, El-Kady MF (2017) Experimental co-infection of infectious bronchitis and low pathogenic avian influenza H9N2 viruses in commercial broiler chickens. Res Vet Sci 115:356-362. https://doi.org/10.1016/j.rvsc.2017.06.024

28. Jiang L, Zhao W, Han Z, Chen Y, Zhao Y, Sun J, Li H, Shao Y, Liu L, Liu S (2017) Genome characterization, antigenicity and pathogenicity of a novel infectious bronchitis virus type isolated from south China. Infect Genet Evol 54:437-446. https://doi. org/10.1016/j.meegid.2017.08.006

29. Yan S, Sun Y, Huang X, Jia W, Xie D, Zhang G (2019) Molecular characteristics and pathogenicity analysis of QX-like avian infectious bronchitis virus isolated in China in 2017 and 2018. Poult Sci. https://doi.org/10.3382/ps/pez351

30. De Wit JJ (2000) Detection of infectious bronchitis virus. Avian Pathol 29(2):71-93. https://doi.org/10.1080/03079450094108

31. Ma H, Shao Y, Sun C, Han Z, Liu X, Guo H, Liu X, Kong X, Liu $S$ (2012) Genetic diversity of avian infectious bronchitis coronavirus in recent years in China. Avian Dis 56(1):15-28. https://doi. org/10.1637/9804-052011-Reg.1

32. Li M, Wang XY, Wei P, Chen QY, Wei ZJ, Mo ML (2012) Serotype and genotype diversity of infectious bronchitis viruses isolated during 1985-2008 in Guangxi, China. Arch Virol 157(3):467-474. https://doi.org/10.1007/s00705-011-1206-6

33. Feng K, Xue Y, Wang F, Chen F, Shu D, Xie Q (2014) Analysis of $\mathrm{S} 1$ gene of avian infectious bronchitis virus isolated in southern China during 2011-2012. Virus Genes 49(2):292-303. https://doi. org/10.1007/s11262-014-1097-1

34. Callison SA, Jackwood MW, Hilt DA (2001) Molecular characterization of infectious bronchitis virus isolates foreign to the United States and comparison with United States isolates. Avian Dis 45(2):492-499

35. Hofstad MS (1981) Cross-immunity in chickens using seven isolates of avian infectious bronchitis virus. Avian Dis 25(3):650-654

36. Huang YP, Wang CH (2007) Sequence changes of infectious bronchitis virus isolates in the $3^{\prime} 7.3 \mathrm{~kb}$ of the genome after attenuating passage in embryonated eggs. Avian Pathol 36(1):59-67. https ://doi.org/10.1080/03079450601110015

37. Casais R, Dove B, Cavanagh D, Britton P (2003) Recombinant avian infectious bronchitis virus expressing a heterologous spike gene demonstrates that the spike protein is a determinant of cell tropism. J Virol 77(16):9084-9089. https://doi.org/10.1128/ jvi.77.16.9084-9089.2003

38. Wickramasinghe IN, de Vries RP, Grone A, de Haan CA, Verheije MH (2011) Binding of avian coronavirus spike proteins to host factors reflects virus tropism and pathogenicity. J Virol 85(17):8903-8912. https://doi.org/10.1128/JVI.05112-11

39. Feng K, Xue Y, Wang J, Chen W, Chen F, Bi Y, Xie Q (2015) Development and efficacy of a novel live-attenuated QX-like nephropathogenic infectious bronchitis virus vaccine in China. Vaccine 33(9):1113-1120. https://doi.org/10.1016/j.vacci ne.2015.01.036

Publisher's Note Springer Nature remains neutral with regard to jurisdictional claims in published maps and institutional affiliations. 